\title{
High Resolution Spatial Modelling Approaches for Monitoring Surface Water and Erosion Impacts of Coal Seam Gas Infrastructure
}

\author{
$\underline{\text { N.I. Huth }}^{\text {a }}$, P.L. Poulton a ${ }^{\text {, P. Caccetta }}{ }^{\text {b }}$, W. Xiaoliang ${ }^{\text {b }}$, B. Cocks ${ }^{\text {a }}$ and J. Wallace ${ }^{\text {b }}$ \\ ${ }^{a}$ CSIRO Agriculture, Toowoomba, Queensland. \\ ${ }^{\text {a } C S I R O ~ D i g i t a l}$ Productivity, Perth, Western Australia.
}

Email:neil.huth@,csiro.au

\begin{abstract}
Coal Seam Gas (CSG) development within the Surat Basin of Queensland, Australia, is expanding, with petroleum leases already approved for tenements covering over $24,000 \mathrm{~km}^{2}$. Many thousands of kilometres of access tracks will be developed to allow transport of vehicles to each of the CSG wells (Figure 1). Studies from agricultural watersheds across the globe have shown that rural roads provide a disproportionate contribution toward sediment loads into water ways. With CSG development, the intensity of roadways in agricultural land will increase significantly and there is a risk that erosion losses will increase likewise. We have evaluated the use of surface water flow models derived from fine-scale digital elevation models for identifying areas where CSG infrastructure has altered surface hydrology and areas of erosion risk.
\end{abstract}

Aerial digital photogrammetry with a ground sample distance of $20 \mathrm{~cm}$ was used to create elevation models for a $1200 \mathrm{~km}^{2}$ focal region currently undergoing CSG development. Baseline digital terrain models were processed using a multi-direction flow-path prediction model. The ground elevation model (GEM) was evaluated using survey data at three different sites (a road cross section, a gravel pad, a road crossing a gully) obtained using a hand held Real-time Kinematic Global Navigation Satellite System (RTK GNSS). The water flow model was assessed using manual recording of visible water paths observed in open agricultural field conditions and within native forests where a significant canopy cover could influence predictions of soil surface elevation.

Comparison of the GEM with ground surface elevation measurements showed good agreement with many of the predictions showing an error of less than $5 \mathrm{~cm}$. This accuracy is approaching that of the RTK GNSS system $( \pm 2 \mathrm{~cm})$ used in the ground surveys. A subset of predictions showed higher levels of error. Investigation found these to predominantly include points where sudden changes in elevation occurred, which suggests error introduced by smoothing algorithms used in creating the GEM. Comparison of predicted water flow paths against those mapped directly indicates that the model is able to accurately identify the location of many water flow paths across roadways. The model was effective in locating the major water flow paths under forest conditions, even when some of the soil surface may be obscured by foliage.

Exploration, interpretation and discussion of the datasets with agricultural landholders and CSG staff developed interest in portraying the information as water flow maps such as those presented here.

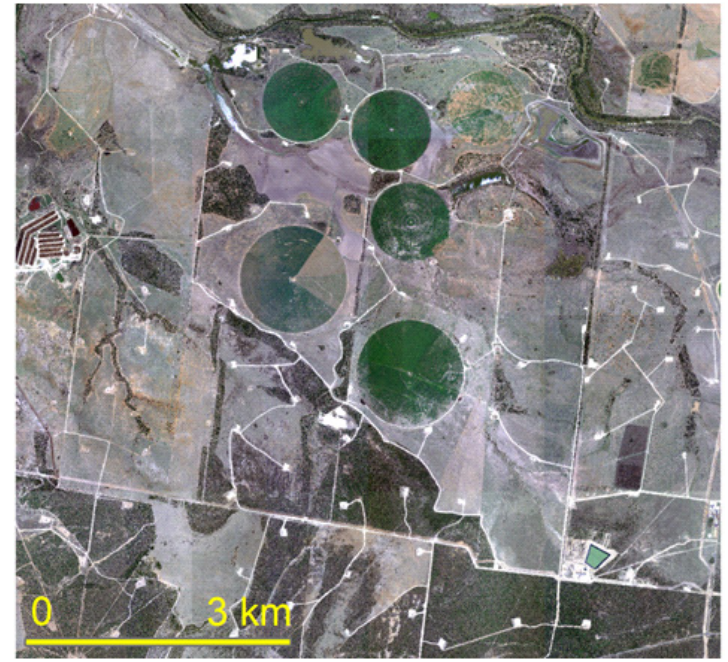

Figure 1. Aerial photograph of the CSG development area, near Condamine, Queensland, showing a network of access tracks connecting CSG well pads.

Keywords: Coal seam gas (CSG), erosion, water flow modelling, digital elevation model (DEM) 
Huth et al., High Resolution Spatial Modelling Approaches for Monitoring Surface Water and Erosion Impacts of Coal Seam Gas Infrastructure

\section{INTRODUCTION}

Much of the Surat Basin in Queensland, Australia, is currently undergoing rapid development for Coal Seam Gas (CSG), with petroleum leases already approved for tenements covering over $24,000 \mathrm{~km}^{2}$ (Huth et al., 2014). Tens of thousands of wells will be installed during the development phase. CSG infrastructure consists of a network of well pads at a density of approximately 1 to 2 per square kilometre. Servicing these wells is a network of access roads, pipelines for gas and water, and electrical power supply. In addition to these is more than $4000 \mathrm{~km}$ of transmission pipelines from the Surat Basin to Gladstone to connect these producing gas fields to local and export markets. The large network of access tracks will be required to cross cultivated, grazing or forested land, presenting an erosion risk.

Studies from around the world have shown that roadways provide a disproportionate source of sediment into waterways. Commonly, over $40 \%$ of sediment in rural runoff originates from unpaved roads, even though these roads make up only about $1-2 \%$ of the total area of a catchment (e.g. Motha et al 2004, Minella et al 2008). With CSG development, the intensity of roadways in agricultural land will increase significantly with a concommitent increase in erosion. Standard engineering methods for mitigating erosion threats are available, if the location of problem areas can be identified. However, the scale of CSG development is so large that monitoring for risk development using traditional methods will be difficult. Therefore, we have investigated the use of photogrammetric approaches for developing fine-scale models of soil surface elevation and water flows for monitoring the impacts of CSG development and for informing planning and land management on the likely locations of erosion risk.

\section{THE STUDY REGION}

The study area is located between the towns of Miles, Condamine and Chinchilla, Queensland, Australia (Figure 2) and covers an area of $36 \mathrm{~km}$ by $36 \mathrm{~km}$ (ie. $1296 \mathrm{~km}^{2}$ ). This area includes several CSG processing plants and the associated large number of CSG wells. Agriculture within the region includes grazing on native and improved pastures, animal feedlots, dry land cropping and irrigated cropping. There are also large areas of native forest.

\section{METHODS}

\subsection{Development of spatial datasets}

Aerial digital photogrammetry techniques were employed in this study to derive the fundamental datasets: a Digital Surface Model (DSM), Ground Elevation Model (GEM) and an orthoimage (Kraus and Pfiefer, 1998). An ADS80 push-broom aerial camera system (Sandau, 2010) was used to capture the aerial imagery on $27^{\text {th }}$ and $28^{\text {th }}$ of November 2013. Images were captured using 22 survey strips (south-north flying direction) and two tie strips

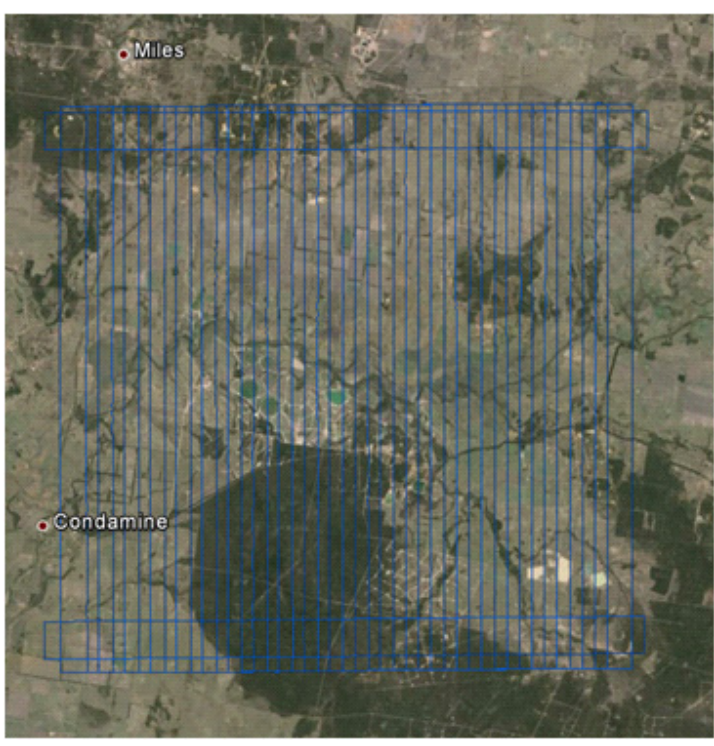

Figure 2. Study area in Queensland, Australia. The blue lines are the outlines of ADS capturing strips. Background image is from Google Earth. (west-east direction) (Figure 2). The ground sample distance (GSD) was $20 \mathrm{~cm}$ for all views (nadir, backward and forward) and all bands (panchromatic band and 4-band spectral bands). The acquired imagery retained the original dynamic range and did not apply a radiometric calibration process. All strips went through a rigorous photogrammetric process including aerial triangulation and generation of the DSM and subsequent orthoimage.

An additional radiometric calibration step was applied to remove differences between the images caused by sun angle, camera view angle and the Bidirectional Reflectance Distribution Function (BRDF) (Collings et al., 2011). Radiometric calibration of the orthoimage is important to classify land cover and therefore automatically track changes in a time-series of images. Painted fibreboard targets $(1.2 \mathrm{~m} \times 1.2 \mathrm{~m})$ were deployed at geographically dispersed sites throughout the study area at the time of data acquisition to facilitate the calibration. Deployment was on flat open ground in sets of three (light grey, dark grey or black) thus providing low-, medium-, and high-reflectance ground truths for the calibration process. The targets were measured in the laboratory with an ASD FieldSpec Pro spectrometer to obtain the absolute reflectance 
values. The calibration was achieved by fitting BRDF kernels (Roujean et al., 1992) designed to model radiometric inconsistencies due to different viewing and illumination angles. The fitting was constrained by enforcing similarity on the overlapping regions between frames $(\mathrm{Wu}, 2006)$ and spatial smoothness of the BRDF coefficients. Additionally, multiplicative (gain) and additive constants were applied to each frame to account for atmospheric and instrument effects.

The aerial triangulation was performed by the company contracted to acquire the aerial imagery using Orima software. The DSM was generated using in-house proprietary software using the approach of Wu (1995). Grid-based relaxation techniques were performed on the object space and reliable matching points were then gridded to form the final DSM. The water flow model used in this analysis requires a model of the ground surface that does not contain the trees, buildings, and other structures in the DSM. This GEM was generated by identifying a set of candidate ground points from the DSM using segmentation, followed by filtering of outliers, then creating a spatially contiguous surface using a thin plate spline surface fitting procedure applied to the candidate ground points (Terzopoulos, 1988).

Finally, predictions of surface water flow paths were generated using the GEM and terrain analysis as described by Caccetta et al. (2010). The predictions are based upon estimates of upslope area, and the maps produced are often referred to water accumulation maps. Surface water flow paths for both $100 \mathrm{~cm}$ and 20 $\mathrm{cm}$ horizontal resolution were generated. Maps of upslope catchment area (Figures 3d, 5a and 5c) have been colour coded for this publication using a logarithmic scale (cyan, $>0.1$ ha; green, $>1$ ha; yellow, $>10$ ha; red, $>100$ ha).

\subsection{Ground measurements for model testing}

Prediction of water flows at very fine spatial scales requires a DEM that is accurate in describing variation in surface elevation at a similar resolution. Three study sites were selected to test the proposed methodology in capturing the surface elevation differences for a range of geographic features of differing size. These included surface variation across a roadway (Site 1), an engineering feature including a raised and compacted gravel foundation for a large water tank (Site 2), and a larger geographic feature including a hill slope, roadway, and some smaller features creating local variation (Site 3). All three sites lie within the farm "Monreagh" about $30 \mathrm{~km}$ WSW of the township of Chinchilla, Queensland.

Site 1 was chosen to provide a detailed study of a large roadway. The road is approximately $10 \mathrm{~m}$ wide and made of compacted gravel. The section of road runs in a NS direction and includes drains on either side to allow water to flow to the south. Seven EW transects across these features were sampled to provide data on the micro-topological variation across this site (e.g. the camber of the road and side drains). This site was chosen to provide data for testing the small-scale variation in elevation across roadways. This is important for uses of the derived model for studying water flow impacts due to roadways and CSG access tracks.

Site 2 includes a large compacted gravel foundation for a large water tank and its surroundings. A drain and contour bank run along the northern edge of the gravel pad to direct water from a large cropping paddock around this structure. Water from this drain breaks through this contour bank at a point within this study area and water flows toward and the western edge of the pad and then flows away from the pad after passing its south-western corner. This site provided data for evaluating the derived surface model for small to medium sized features in agricultural landscapes.

Site 3 comprises a larger area which, includes a range of features of differing size. An access track runs NS along the eastern edge of the site and this crosses a gully running EW. Ground to the west of this has been used for the installation of a large buried pipeline. Several small contour banks have been installed onto the soil surface above the pipeline to manage water flow. In several locations, water from the contour banks is fed into the roadside drain on the western side of the road. In other locations, water appeared to flow across the road. This study site was chosen to provide data on the ability of the derived surface elevation model to capture both the smaller and larger scale features in these agricultural landscapes.

Ground surveys of surface elevation were undertaken at each field site on the 3rd of December 2013 using a Real-time Kinematic Global Navigation Satellite System (RTK GNSS). The tolerance range for these measurements is expected to be $\pm 2 \mathrm{~cm}$. Individual height measurements were selected to capture the range of elevation within each study site and local site variation around various features within each site. Such features included anthropogenic structures such as roads, drains, runoff contour banks and pads, and hydrologically formed features such as erosion rills and gullies.

These individual elevation measurements were collected to enable testing of the precision and accuracy of the surface elevation models developed using the photogrammetric methods. A set of statistical measures of 
Huth et al., High Resolution Spatial Modelling Approaches for Monitoring Surface Water and Erosion Impacts of Coal Seam Gas Infrastructure

model effectiveness were calculated. These included Nash-Sutcliffe Efficiency (NSE) and root mean square error to standard deviation ratio (RSR) to express model error relative to data variance (Moriasi et al., 2007) and Mean Error (ME) and Root Mean Square Error (MAE) to show bias and average error in simple length terms.

Finally, mapping of visually identified water flow paths was undertaken at nine locations within the study region. Sites included open agricultural areas and native woodlands with medium levels of canopy cover. Recorded flow paths ranged from 9 to $498 \mathrm{~m}$ in length. Surface water flow paths and erosion rills were walked and mapped using a differential GPS with $\pm 30 \mathrm{~cm}$ horizontal accuracy. These were compared graphically with surface flow paths modelled at $20 \mathrm{~cm}$ and $100 \mathrm{~cm}$ horizontal resolution from the aerial imagery.

\section{RESULTS}

a) RGB image from aerial survey

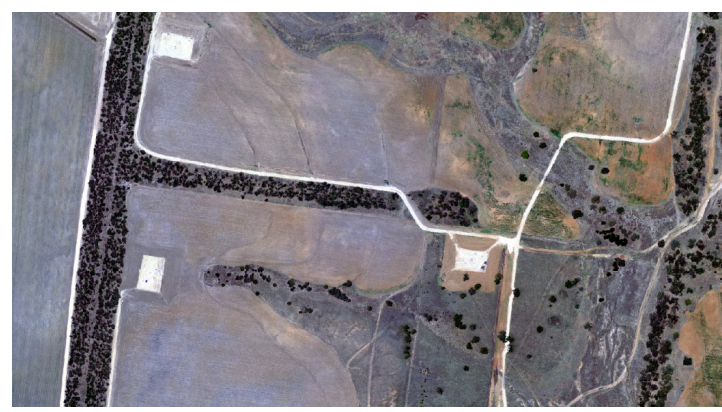

c) GEM (trees and buildings removed) b) DSM including trees and buildings

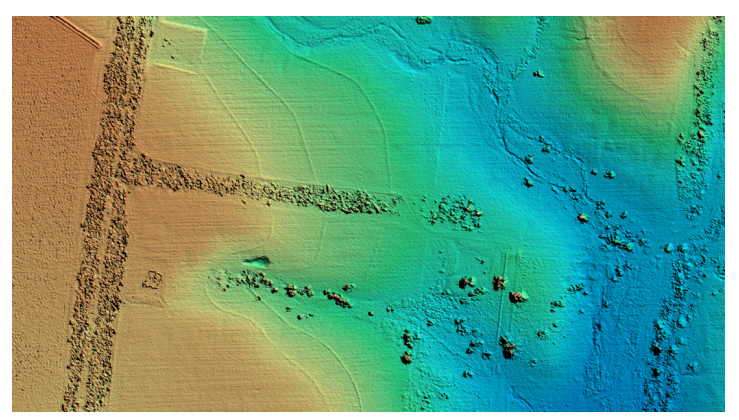

d) Water flow model derived from the GEM.

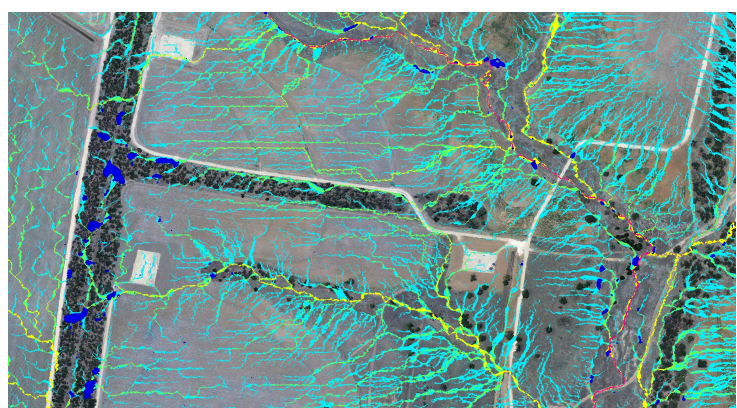

Figure 3. (a) RGB image from aerial photogrammetry. (b) Digital surface model (DSM) at $20 \mathrm{~cm}$ resolution highlighting trees and surface infrastructure. (c) Generated ground elevation model (GEM) after removal of above ground structures. (d) Modelled accumulated flow paths indicating low (green) to high (red) accumulation overlaid on RGB image.

\subsection{Development of the spatial datasets}

Examples of the spatial data and the information provided by each are shown in Figure 3. These images cover an area which includes three CSG wells and their related access tracks and buried pipelines. Note that the location of contour banks within cultivated areas are easily identified, as are the locations of natural surface depressions within remnant vegetation once the trees are removed to generate the GEM.

\subsection{Evaluation of the Digital Elevation Model}

The accuracy of the generated GEM was evaluated using the ground survey data from the 3 detailed study sites. Comparison of the predicted elevation against field point measurements showed a small but consistent bias at each of the 3 study sites $(10.7 \mathrm{~cm}, 10 \mathrm{~cm}$ and $21.1 \mathrm{~cm}$ for sites 1,2 and 3 respectively). This local bias was corrected before evaluating the DEM at each site given our interest in fine-scale water flow predictions. Table 1 shows various statistical indicators of the DEM's accuracy in predicting variation in elevation across the three study sites. The table also includes box plots of the error distribution across all the survey points at each site. The GEM successfully captured small-scale variation at each site. MAE was 
Huth et al., High Resolution Spatial Modelling Approaches for Monitoring Surface Water and Erosion Impacts of Coal Seam Gas Infrastructure

small at each site, especially when compared to the measurement error inherent in the measurements $(\mathrm{c} . \pm 2 \mathrm{~cm})$. NSE and RSR describe model error relative to variance in the observed data. Hence, it is not surprising to see better values for these two statistics for sites with larger range in elevation. Values of RMSE were influenced by a small number of points with larger errors at each site (Table 1). Investigation of these points found them to predominantly include measurements where sudden changes in elevation occurred (e.g. earth works or gullies) which suggests error introduced by smoothing algorithms used in creating the GEM. This is evident in the data from Site 3 (Figure 4) where the error in the predictions of point elevation measurements were greatest at the base of the gully where ground elevation changed abruptly due to erosion of the water course. Predictions across the rest of Site 3, including those for roads, contour banks, and small drains were mostly accurate to within a few centimetres.
Table 2. Statistical evaluation of the model predictions of elevation at each of the survey points for the three study sites.

\begin{tabular}{lccc}
\hline & $\begin{array}{c}\text { Site 1 } \\
(\mathrm{n}=110)\end{array}$ & $\begin{array}{c}\text { Site 2 } \\
(\mathrm{n}=221)\end{array}$ & $\begin{array}{c}\text { Site 3 } \\
(\mathrm{n}=684)\end{array}$ \\
\hline NSE & 0.858 & 0.835 & 0.996 \\
$\operatorname{RMSE}(\mathrm{m})$ & 0.079 & 0.113 & 0.084 \\
$\operatorname{RSR}$ & 0.375 & 0.279 & 0.057 \\
MAE (m) & 0.064 & 0.081 & 0.052
\end{tabular}

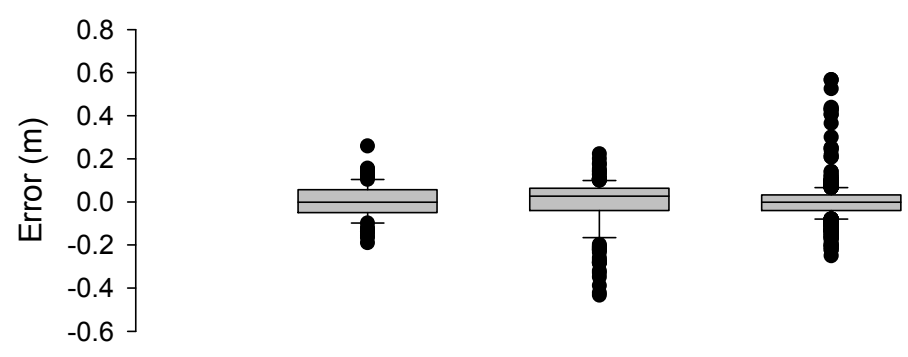

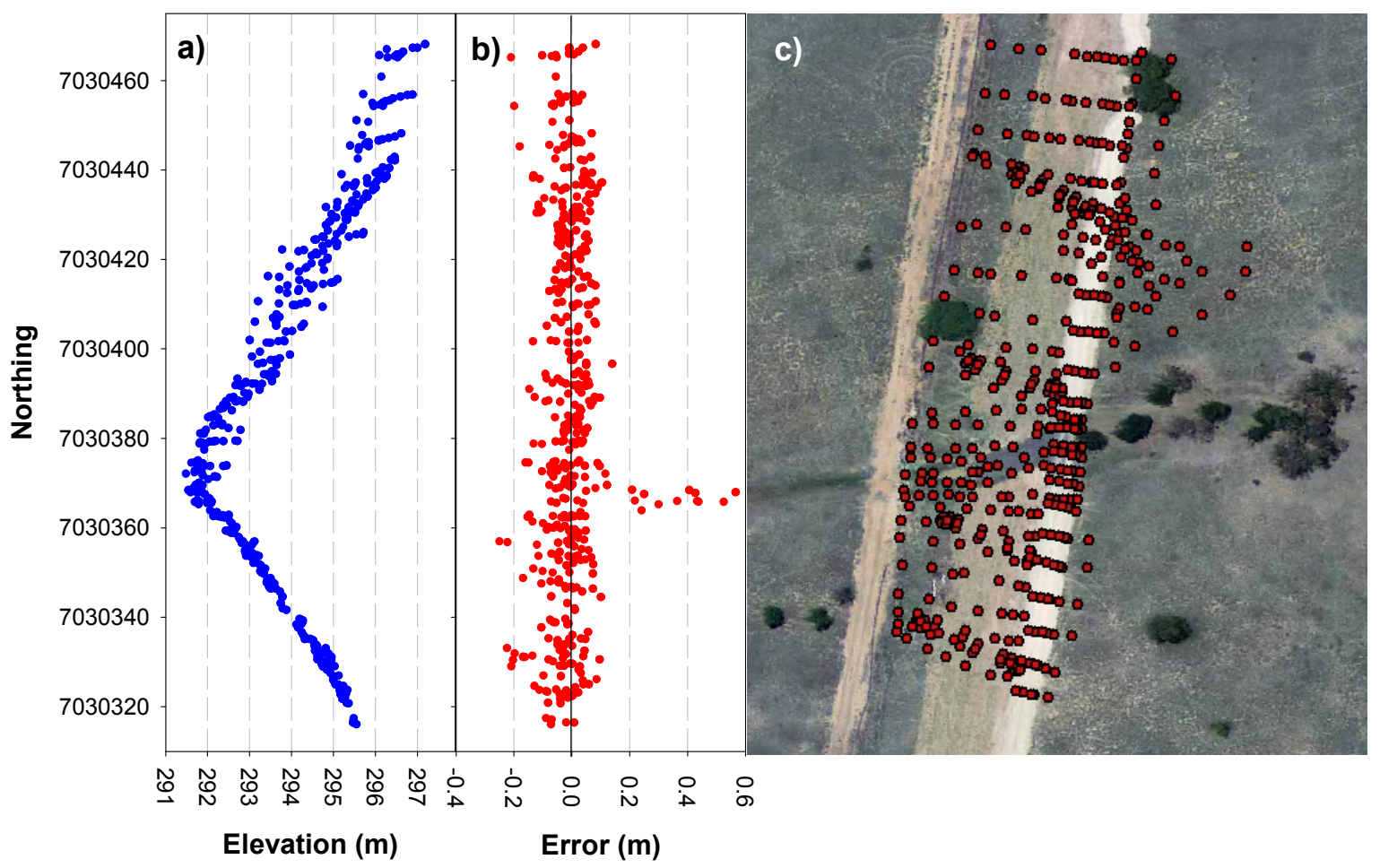

Figure 4. Plot of survey data and model predictions showing a) the location and values of individual elevation measurements used in model testing, b) errors in those model predictions, and c) the locations of the survey measurement locations. 
Huth et al., High Resolution Spatial Modelling Approaches for Monitoring Surface Water and Erosion Impacts of Coal Seam Gas Infrastructure

\subsection{Evaluation of the Water Flow Model}

Evaluation of the water flow paths traced manually in the field showed good agreement in both agricultural and natural settings (Figure 2). Water flow paths across and along farm roads and CSG access tracks were simulated with some success (data not shown) but there was some uncertainty in the observations given the ongoing changes in road surfaces due to traffic, road maintenance and natural erosion processes. However, in many cases the model could accurately identify the resulting change in water flow path and likely location of rill formation where CSG access tracks interrupted runoff water from agricultural fields (Figures 5a and 5b). Furthermore, the ability of the model to predict the location of water flows did not appear to be significantly adversely affected by the presence of tree cover in the native woodland areas within the study region (Figures $5 \mathrm{c}$ and $5 \mathrm{~d}$ ). This suggests that adequate information regarding the soil surface elevation can be obtained with this level of canopy cover.

\section{DISCUSSION AND CONCLUSIONS}

The testing of photogrammetric techniques for the development of ground elevation and water flow models in this study has shown great promise with levels of precision accurate enough for the study of water flows in rural landscapes and relatively fine scales. The level of precision found here is similar to that obtained using photogrammetric approaches on much smaller study areas ( $<10 \mathrm{ha})$ using kites, blimps or unmanned aerial vehicles (Marzolff and Poesen, 2009; Gimenez et al., 2009; Harwin and Lucieer, 2012;) to investigate relatively large erosion features such as gullies. However, this study has performed a similar analysis over a much larger area (i.e. $1296 \mathrm{~km}^{2}$ ) to investigate surface water flows and subtle changes in surface topography due to the addition of CSG access tracks.

Discussion and interpretation of the datasets with agricultural land holders and CSG staff generated interest in the information portrayed in the water flow maps. Information on the location and catchment area of water flows can be used by land holders and CSG staff during planning for CSG infrastructure placement. Furthermore, repeated surveys can show changes in water flow or soil surface elevation, which may indicate diversion of water flows, soil loss, or build-up of sediment within the survey area. The likely source of any

a) Flow paths on upslope of track

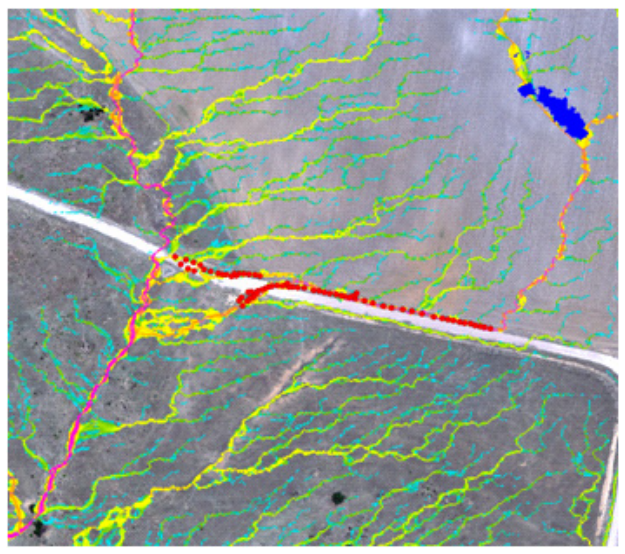

c) Flow path through native forest

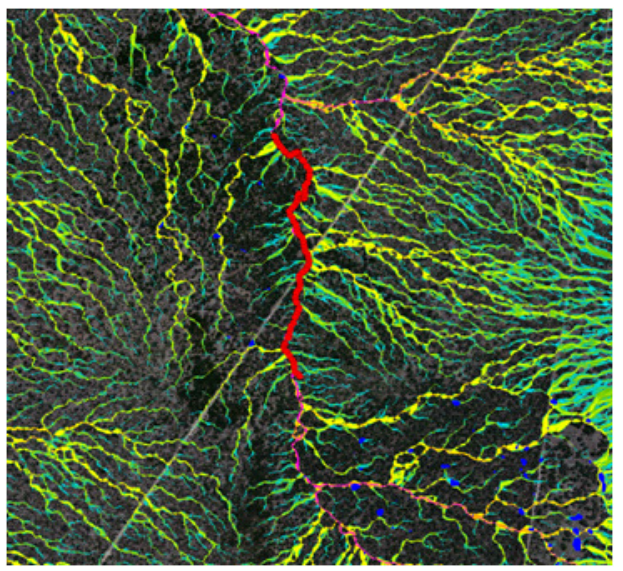

b) Photo of developing rill at this location

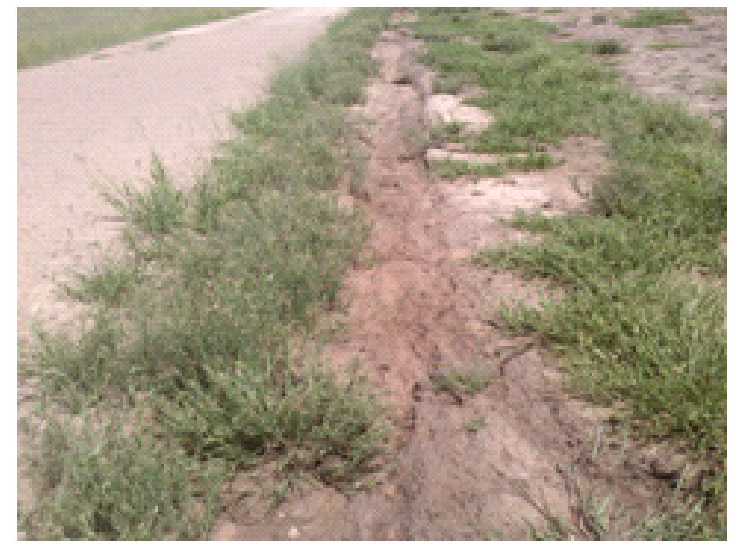

d) Photo of survey of along water course

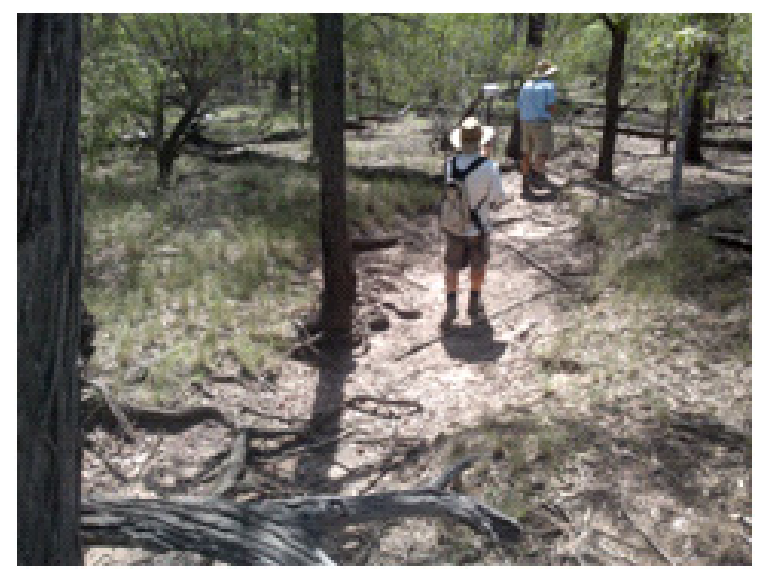

Figure 5. Predicted (lines), measured DGPS locations (red dots) and photographs of flow lines for a developing rill next to a CSG access track $(a, b)$, and a natural water course in native forest $(c, d)$. 
Huth et al., High Resolution Spatial Modelling Approaches for Monitoring Surface Water and Erosion Impacts of Coal Seam Gas Infrastructure

sediment build-up can be identified by following the predicted water flow paths to locations of change in surface elevation. Furthermore, concerns by land holders regarding surface water flows can be better communicated with the use of water flow maps. These are just a few examples of how these data can better facilitate management, monitoring, and communication of erosion risks as these landscapes continue to become highly modified.

\section{ACKNOWLEDGMENTS}

The Gas Industry Social and Environmental Research Alliance (GISERA) undertake publicly-reported research that addresses the socio-economic and environmental impacts of Australia's natural gas industries. GISERA was co-founded by CSIRO and Australia Pacific LNG in July 2011. In March 2014, QGC joined GISERA in recognition that the challenges being addressed through GISERA are relevant to the entire CSGLNG industry, and that there are benefits from such collaborative research. For further information please visit: www.gisera.org.au.

\section{REFERENCES}

Caccetta, P.A., Dunne, R., George, R. and McFarlane, D. (2010). A Methodology to Estimate the Future Extent of Dryland Salinity in the Southwest of Western Australia. Journal of Environmental Quality, 39:26-34.

Collings, S., Caccetta, P.A., Campbell, N.A. and Wu, X. (2011). Empirical Models for Radiometric Calibration of Digital Aerial Frame Mosaics, IEEE Transactions on Geoscience and Remote Sensing, 49(7):2573-2588.

Gimenez, R., Marzolff, I., Campo, M.A., Seeger, M., Ries, J.B., Casali, J. and Alvarez-Mozos, J. (2009). Accuracy of high-resolution photogrammetric measurements of gullies with contrasting morphology. Earth Surface Processes and Landforms, 34, 1915-1926.

Harwin, S. and Lucieer, A. (2012). Assessing the Accuracy of Georeferenced Point Clouds Produced via Multi-View Stereopsis from Unmanned Aerial Vehicle (UAV) Imagery. Remote Sensing, 4, 1573-1599.

Huth N.I., Cocks B., Dalgliesh N., Poulton, P., Marinoni, O., and Navarro J. (2014). Farmers' perceptions of coexistence between agriculture and large scale coal seam gas development: working paper, June 2014, CSIRO, Australia. ISBN (online): 978-1-4863-0362-5.

Kraus, K. and Pfeifer, N. (1998). Determination of terrain models in wooded areas with airborne laser scanner data, ISPRS Journal of Photogrammetry and Remote Sensing, 53, 193-203.

Marzolff, I. and Poesen, J. (2009). The potential of 3D gully monitoring with GIS using high-resolution aerial photography and a digital photogrammetry system. Geomorphology, 111, 48-60.

Minella, J.P.G., Walling, D.E., and Merten, G.H. (2008). Combining sediment source tracing techniques with traditional monitoring to assess the impact of improved land management on catchment sediment yields. Journal of Hydrology, 348, 546-563.

Moriasi, D.N., Arnold, J.G., Van Liew, M.W., Bingner, R.L. and Harmel, R.D. (2007). Model evaluation guidelines for systematic quantification of accuracy in watershed simulations. Transactions of the ASAE, $50,885-900$.

Motha, J.A., Wallbrink, P.J., Hairsine, P.B., and Grayson, R.B. (2004). Unsealed roads as suspended sediment sources in an agricultural catchment in south-eastern Australia. Journal of Hydrology, 286, 1-18.

Roujean, J. L., Leroy, M. and Deshchamps, P.Y. (1992). A bidirectional reflectance model of the earth's surface for the correction of remote sensor data, Journal of Geophysical Research, 97(D18): 455-468.

Sandau, R. (Ed.) (2010). Digital Airborne Camera: Introduction and Technology, Springer.

Terzopoulos, D. (1988). The computation of visible-surface representations, IEEE Transactions on Pattern Analysis and Machine Intelligence, 10(4): 417 - 438.

Wu, X. (1995). Grey-based Relaxation for Image Matching. DICTA-95, 3rd Conference on Digital Image Computing: Techniques and Applications, December, 1995, Brisbane, Australia.

Wu, X. (2006). Radiometric Calibration of Digital Aerial Imagery, Proceedings of the 13th Australian Remote Sensing and Photogrammetry Conference, Canberra, Australia. 